\title{
INTERPOLATIONS OF DETERMINANTAL INEQUALITIES OF JENSEN'S TYPE
}

\author{
DRAGOSLAV S. MITRINOVIĆ AND JOSIP E. PEČARIĆ
}

As in [1] and [2] we shall use the following notation:

$\mathcal{M}=\{M \mid M$ is a positive definite matrix of order $n\}$,

$|M|=$ the determinant of the matrix $M$,

$|M|_{k}=\Pi_{j=1}^{k} \lambda_{j}, k=1, \cdots, n$, where $\lambda_{1}, \ldots, \lambda_{n}$ are the eigenvalues of $M$ with $\lambda_{1} \leq \ldots \leq$ $\lambda_{n},|M|_{n}=|M|$,

$M(j)=$ the submatrix of $M$ obtained by deleting the $j^{\text {th }}$ row and column of $M$,

$M[k]=$ the principal submatrix of $M$ formed by taking the first $k$ rows and columns of $M, M[n]=M, M[n-1]=M(n), M[0]=$ the identity matrix,

$\mathrm{BBF}=$ the class of Bellman-Bergstrom-Fan quasi-linear functionals $\sigma_{i}, \delta_{j}$, and $\nu_{k}$ defined on $\mathcal{M}$ by

$$
\begin{gathered}
\sigma_{i}(M)=|M|_{i}^{1 / i}, i=1, \cdots, n, \\
\delta_{j}(M)=|M| /|M(j)|, j=1, \cdots, n,
\end{gathered}
$$

and

$$
\nu_{k}(M)=(|M| /|M[k]|)^{1 /(n-k)}, k=1, \cdots, n,
$$

respectively. It is evident that $\mathcal{M}$ is closed under addition and multiplication by a positive number, i.e. if $M_{1}, M_{2} \in \mathcal{M}, a>0$, then

$$
M_{1}+M_{2}, a M_{1} \in \mathcal{M}
$$

Now, quasi-linearity of the BBF functionals follows from results given in [3, pp. 67 , $70,71]$ (see also $[1,2]$ ), i.e.

$$
\phi\left(p M_{1}+q M_{2}\right) \geq p \phi\left(M_{1}\right)+q \phi\left(M_{2}\right)
$$

for $M_{1}, M_{2} \in \mathcal{M}, p, q>0, \phi \in B B F$.

More generaly, for $M_{i} \in \mathcal{M}, p_{i}>0(i=1, \cdots, m), P_{k}=\Sigma_{i=1}^{k} p_{i}(k=1, \cdots, m), \phi \in$ $B B F$, we have [2]:

$$
\phi\left(\sum_{i=1}^{m} p_{i} M_{i}\right) \geq \sum_{i=1}^{m} p_{i} \phi\left(M_{i}\right) \geq P_{m} \prod_{i=1}^{m} \phi\left(M_{i}\right)^{p_{i} / P_{m}}
$$

Received October 12, 1989. 
what is an interpolating inequality for

$$
\phi\left(\frac{1}{P_{m}} \sum_{i=1}^{m} p_{i} M_{i}\right) \geq \prod_{i=1}^{m} \phi\left(M_{i}\right)^{p_{i} / P_{m}} .
$$

Note that (2) is also a generalization of a result from [4].

For $p_{i}=1 / m,(1)$ and (2) become

$$
\phi\left(\frac{1}{m} \sum_{i=1}^{m} M_{i}\right) \geq \frac{1}{m} \sum_{i=1}^{m} \phi\left(M_{i}\right) \geq \prod_{i=1}^{m} \phi\left(M_{i}\right)^{1 / m}
$$

and

$$
\phi\left(\frac{1}{m} \sum_{i=1}^{m} M_{i}\right) \geq \prod_{i=1}^{m} \phi\left(M_{i}\right)^{1 / m}
$$

Here, we shall give some interpolations of inequalities (3) and (4). In there results we shall use the following expresions:

$$
\begin{gathered}
f_{k, m}=\frac{1}{\left(\begin{array}{c}
m \\
k
\end{array}\right)} \sum_{1 \leq i_{1}<\ldots<i_{k} \leq m} \phi\left(\frac{1}{k}\left(M_{i_{1}}+\cdots+M_{i_{k}}\right)\right), \\
g_{k, m}=\prod_{1 \leq i_{1}<\ldots<i_{k} \leq m}\left(\frac{1}{k}\left(\phi\left(M_{i_{1}}\right)+\cdots+\phi\left(M_{i_{k}}\right)\right)\right)^{1 /\left(\begin{array}{c}
m \\
k
\end{array}\right)} \\
h_{k, m}=\frac{1}{\left(\begin{array}{c}
m \\
k
\end{array}\right)} \sum_{1 \leq i_{1}<\ldots<i_{k} \leq m}\left(\phi\left(M_{i_{1}}\right) \cdots \phi\left(M_{i_{k}}\right)\right)^{1 / k} \\
r_{k, m}=\prod_{1 \leq i_{1}<\ldots<i_{k} \leq m} \phi\left(\frac{1}{k}\left(M_{i_{1}}+\ldots+M_{i_{k}}\right)\right)^{1 /\left(\begin{array}{c}
m \\
k
\end{array}\right) .} .
\end{gathered}
$$

First, we shall prove the following interpolations of the first inequality in (3):

$$
\phi\left(\frac{1}{m} \sum_{i=1}^{m} M_{i}\right)=f_{m, m} \geq \ldots \geq f_{k+1, m} \geq f_{k, m} \geq \ldots \geq f_{1, m}=\frac{1}{m} \sum_{i=1}^{m} \phi\left(M_{i}\right) .
$$

Proof. We have

$$
\begin{aligned}
f_{k+1, m} & =\frac{1}{\left(\begin{array}{c}
m \\
k+1
\end{array}\right)} \sum_{1 \leq i_{1}<\cdots<i_{k+1} \leq m} \phi\left(\frac{M_{i_{1}}+\cdots+M_{i_{k+1}}}{k+1}\right) \\
& \geq \frac{1}{\left(\begin{array}{c}
m \\
k+1
\end{array}\right)} \sum_{1 \leq i_{1}<\cdots<i_{k+1} \leq m} \frac{1}{k+1} \sum_{j=1}^{k+1} \phi\left(\frac{M_{1_{1}}+\cdots+M_{i_{k+1}}-M_{i_{j}}}{k}\right) \\
& =\frac{1}{\left(\begin{array}{c}
m \\
k
\end{array}\right)} \sum_{1 \leq i_{1}<\cdots<i_{k} \leq m} \phi\left(\frac{M_{i_{1}}+\cdots+M_{i_{k}}}{k}\right)=f_{k, m} .
\end{aligned}
$$


The second inequality in (3) has the following interpolations:

$$
\begin{aligned}
& \frac{1}{m} \sum_{i=1}^{m} \phi\left(M_{i}\right)=g_{m, m} \geq \ldots \geq g_{k+1, m} \geq g_{k, m} \geq \ldots \geq g_{1, m}=\prod_{i=1}^{m} \phi\left(M_{i}\right)^{1 / m}, \\
& \frac{1}{m} \sum_{i=1}^{m} \phi\left(M_{i}\right)=h_{1, m} \geq \ldots \geq h_{k, m} \geq h_{k+1, m} \geq \ldots \geq h_{m, m}=\prod_{i=1}^{m} \phi\left(M_{i}\right)^{1 / m} .
\end{aligned}
$$

Proof. Now, we have

$$
\begin{aligned}
g_{k+1, m} & =\prod_{1 \leq i_{1}<\ldots<i_{k+1} \leq m}\left(\frac{\phi\left(M_{i_{1}}\right)+\ldots+\phi\left(M_{i_{k+1}}\right)}{k+1}\right)^{1 /\left(\begin{array}{c}
m \\
k+1
\end{array}\right)} \\
& \geq \prod_{1 \leq i_{1}<\ldots<i_{k+1} \leq m}\left(\prod_{i=1}^{k+1}\left(\frac{\phi\left(M_{i_{1}}\right)+\ldots+\phi\left(M_{i_{k+1}}\right)-\phi\left(M_{i_{j}}\right)}{k}\right)^{\frac{1}{k+1}}\right)^{\frac{1}{\left(\begin{array}{c}
m \\
k+1
\end{array}\right)}} \\
& =\prod_{1 \leq i_{1}<\ldots<i_{k} \leq m}\left(\frac{\phi\left(M_{i_{1}}\right)+\ldots+\phi\left(M_{i_{k}}\right)}{k}\right)^{1 /\left(\begin{array}{c}
m \\
k
\end{array}\right)}=g_{k, m},
\end{aligned}
$$

and

$$
\begin{aligned}
h_{k+1, m} & =\frac{1}{\left(\begin{array}{c}
m \\
k+1
\end{array}\right)} \sum_{1 \leq i_{1}<\ldots<i_{k+1} \leq m}\left(\phi\left(M_{i_{1}}\right) \ldots \phi\left(M_{i_{k+1}}\right)\right)^{1 /(k+1)} \\
& \leq \frac{1}{\left(\begin{array}{c}
m \\
k+1
\end{array}\right)} \sum_{1 \leq i_{1}<\ldots<i_{k+1} \leq m} \frac{1}{k+1} \sum_{j=1}^{k+1}\left(\frac{\phi\left(M_{i_{1}}\right) \ldots \phi\left(M_{i_{k+1}}\right)}{\phi\left(M_{i_{j}}\right)}\right)^{1 / k} \\
& =\frac{1}{\left(\begin{array}{c}
m \\
k
\end{array}\right)} \sum_{1 \leq i_{1}<\ldots<i_{k} \leq m}\left(\phi\left(M_{i_{1}}\right) \ldots \phi\left(M_{i_{k}}\right)\right)^{1 / k}=h_{k, m} .
\end{aligned}
$$

Finally, similar interpolation of (4) is:

$$
\phi\left(\frac{1}{m} \sum_{i=1}^{m} M_{i}\right)=r_{m, m} \geq \ldots \geq r_{k+1, m} \geq r_{k, m} \geq \ldots \geq r_{1, m}=\prod_{i=1}^{m} \phi\left(M_{i}\right)^{1 / m} .
$$

Proof

$$
\begin{aligned}
r_{k+1, m} & =\prod_{1 \leq i_{1}<\ldots<i_{k+1} \leq m} \phi\left(\frac{M_{i_{1}}+\ldots+M_{i_{k+1}}}{k+1}\right)^{1 /\left(\begin{array}{c}
m \\
k+1
\end{array}\right)} \\
& \geq \prod_{1 \leq i_{1}<\ldots<i_{k+1} \leq m}\left(\prod_{j=1}^{k+1} \phi\left(\frac{M_{i_{1}}+\ldots M_{i_{k+1}}-M_{i_{j}}}{k}\right)^{\frac{1}{k+1}}\right)^{\frac{1}{\left(\begin{array}{c}
m \\
k+1
\end{array}\right)}} \\
& =\prod_{1 \leq i_{1}<\ldots<i_{k} \leq m} \phi\left(\frac{1}{k}\left(M_{i_{1}}+\ldots+M_{i_{k}}\right)\right)^{1 /\left(\begin{array}{c}
m \\
k
\end{array}\right)}=r_{k, m} .
\end{aligned}
$$




\section{References}

[1] D.S. Mitrinović and J.E. Pečarić, "Determinantal inequalities of Jensen's type," Anz. Osterr. Akad. Wiss. math. -naturwiss. Kl. 125 (1988),75-78.

[2] C.L. Wang, "Extensions of determinantal inequalities," Utilitas Math. 13 (1978), 201-210.

[3] E.F. Beckenbach and R. Bellman, Inequalities, Berlin-Heidelberg-New York, 1971.

[4] L. Mirsky, "An inequality for positive definite matrices," Amer. Math. Monthly 62 (1955), 428-430.

D.S. Mitrinović

Smiljanićeva 38, 11000 Beograd, Yugoslavia.

J.E. Pečarić

Faculty of Technology, Ive Lole Ribara 126, 41000 Zagreb, Yugoslavia. 


\title{
GENERALIZATIONS OF OPIAL-TYPE INEQUAIITIES IN TWO VARIABLES
}

\author{
WING-SUM CHEUNG
}

\begin{abstract}
In this paper some generalizations of the existing Opial-type inequalities in two variables are established. Such integral inequalities have a wide range of applications in the study of differential and integral equations.
\end{abstract}

\section{Introduction}

The Opial-type inequalities are among a handful of celebrated integral inequalities which plays an important role in the study of many differential and integral equations. The first Opial-type inequality is the following one obtained by Opial.

Theorem ([5]). If $f \in \mathcal{L}^{1}[0, h]$ satisfies $f(0)=f(h)=0$ and $f(x)>0$ for all $x \in(0, h)$, then

$$
\int_{0}^{h}\left|f(x) f^{\prime}(x)\right| d x \leq \frac{h}{4} \int_{0}^{h}\left|f^{\prime}(x)\right|^{2} d x .
$$

Opial's result has been generalized to many different situations, most of which are for the case of 1 variable ( $\dot{\mathrm{g}}$; [2], [4], [7], [8]). In [3], several Opial-type inequalities in 2 variables have been established. In this paper, we shall further generalize the results obtained in [3] to more general situations. For other Opial-type inequalities in 2-variables, which are less general, one is referred to, e.g., [6], and the references cited there.

\section{Main Results}

In what follows we shall assume that $f, g, f_{1}, g_{1}, f_{12}$, and $g_{12}$ are continuous realvalued functions on $[a, b] \times[c, d]$, where, as usual, the subscripts refer to partial derivatives. Let $w$ be a positive continuous weight function on $[a, b] \times[c, d]$. Let $r$ be any positive function on $[a, b] \times[c, d]$ with $r^{-1} \in \mathcal{L}^{1}([a, b] \times[c, d])$.

Theorem 1 . If $f(a, t)=g(a, t)=f_{1}(s, c)=g_{1}(s, c)=0$ for all $(s, t) \in[a, b] \times[c, d]$ 
and if $w(s, t)$ is nonincreasing in each variable, then

$$
\begin{aligned}
& \int_{a}^{b} \int_{c}^{d} w|f g|^{p}\left(\left|f g_{12}\right|^{q}+\left|f_{12} g\right|^{q}\right) d t d s \\
\leq & \frac{q}{2(p+q)}[(b-a)(d-c)]^{2 p+q-1} \cdot \int_{a}^{b} \int_{c}^{d} r^{-1} d t d s . \\
& \int_{a}^{b} \int_{c}^{d} r w\left(\left|f_{12}\right|^{2(p+q)}+\left|g_{12}\right|^{2(p+q)}\right) d t d s
\end{aligned}
$$

for any real numbers $p \geq 0, q \geq 1$.

Theorem 2. If $f(a, t)=g(a, t)=f_{1}(s, c)=g_{1}(s, c)=0$ and $0<A \leq w(s, t) \leq B$ for all $(s, t) \in[a, b] \times[c, d]$, then

$$
\begin{aligned}
& \int_{a}^{b} \int_{c}^{d} w|f g|^{p}\left(\left|f g_{12}\right|^{q}+\left|f_{12} g\right|^{q}\right) d t d s \\
\leq & \frac{q}{2(p+q)}[(b-a)(d-c)]^{2 p+q-1}\left(\frac{B}{A}\right)^{(2 p+q) /(2(p+q))} \cdot \int_{a}^{b} \int_{c}^{d} r^{-1} d t d s \\
& \times \int_{a}^{b} \int_{c}^{d} r w\left(\left|f_{12}\right|^{2(p+q)}+\left|g_{12}\right|^{2(p+q)}\right) d t d s
\end{aligned}
$$

for any real numbers $p \geq 0, q \geq 1$.

Theorem 3. If $f(a, t)=g(a, t)=f(b, t)=g(b, t)=f_{1}(s, c)=g_{1}(s, c)=f_{1}(s, d)=$ $g_{1}(s, d)=0$ and $0<A \leq w(s, t) \leq B$ for all $(s, t) \in[a, b] \times[c, d]$, then

$$
\begin{aligned}
& \int_{a}^{b} \int_{c}^{d} w|f g|^{p}\left(\left|f g_{12}\right|^{q}+\left|f_{12} g\right|^{q}\right) d t d s \\
\leq & \frac{q}{2(p+q)}\left[\frac{(b-a)(d-c)}{4}\right]^{2 p+q-1}\left(\frac{B}{A}\right)^{(2 p+q) /(2(p+q))} \cdot M \\
& \times \int_{a}^{b} \int_{c}^{d} r w\left(\left|f_{12}\right|^{2(p+q)}+\left|g_{12}\right|^{2(p+q)}\right) d t d s
\end{aligned}
$$

for any real numbers $p \geq 0, q \geq 1$, where

$$
\begin{aligned}
M:=\max & \left\{\int_{a}^{(a+b) / 2} \int_{c}^{(c+d) / 2} r^{-1} d t d s, \int_{a}^{(a+b) / 2} \int_{(c+d) / 2}^{d} r^{-1} d t d s,\right. \\
& \left.\int_{(a+b) / 2}^{b} \int_{c}^{(c+d) / 2} r^{-1} d t d s, \int_{(a+b) / 2}^{b} \int_{(c+d) / 2}^{d} r^{-1} d t d s\right\}
\end{aligned}
$$

Before proving these theorems, we first define, for any $(s, t) \in[a, b] \times[c, d]$,

$$
F(s, t):=\int_{a}^{s} \int_{c}^{t} w(u, v)^{q /(2(p+q))}\left|f_{12}(u, v)\right|^{q} d v d u
$$


and

$$
G(s, t):=\int_{a}^{s} \int_{c}^{t} w(u, v)^{q /(2(p+q))}\left|g_{12}(u, v)\right|^{q} d v d u .
$$

Clearly we have $F \geq 0, G \geq 0$.

$\mathbb{L}$ emma 1 . For any $(s, t) \in[a, b] \times[c, d]$ and any real numbers $p \geq 0, q>0$.

$$
\begin{aligned}
& \int_{a}^{s} \int_{c}^{t} w^{q /(2(p+q))}(F G)^{p / q}\left[F\left|g_{12}\right|^{q}+G\left|f_{12}\right|^{q}\right] d v d u \\
\leq & \frac{q}{2(p+q)}[(s-a)(t-c)]^{2 p / q} \int_{a}^{s} \int_{c}^{t} r^{-1} d v d u \\
& \times \int_{a}^{s} \int_{c}^{t} r w\left(\left|f_{12}\right|^{2(p+q)}+\left|g_{12}\right|^{2(p+q)}\right) d v d u .
\end{aligned}
$$

Proof: Since

$$
\begin{aligned}
& F_{1}(s, t)=\int_{c}^{t} w(s, v)^{q /(2(p+q))}\left|f_{12}(s, v)\right|^{q} d v, \\
& G_{1}(s, t)=\int_{c}^{t} w(s, v)^{q /(2(p+q))}\left|g_{12}(s, v)\right|^{q} d v, \\
& F_{2}(s, t)=\int_{a}^{s} w(u, t)^{q /(2(p+q))}\left|f_{12}(u, t)\right|^{q} d u, \\
& G_{2}(s, t)=\int_{a}^{s} w(u, t)^{q /(2(p+q))}\left|g_{12}(u, t)\right|^{q} d u,
\end{aligned}
$$

both $F$ and $G$ are nondecreasing in each variable. Hence

$$
\begin{aligned}
& \int_{a}^{s} \int_{c}^{t} w(u, v)^{q /(2(p+q))}[F(u, v) G(u, v)]^{p / q} . \\
& {\left[F(u, v)\left|g_{12}(u, v)\right|^{q}+G(u, v)\left|f_{12}(u, v)\right|^{q}\right] d v d u } \\
\leq & \int_{a}^{s}[F(u, t) G(u, t)]^{p / q}\left[F(u, t) G_{1}(u, t)+F_{1}(u, t) G(u, t)\right] d u \\
= & \frac{q}{p+q} \int_{a}^{s}\left[F(u, t)^{(p+q) / q} G(u, t)^{(p+q) / q}\right]_{1} d u \\
= & \frac{q}{p+q} F(s, t)^{(p+q) / q} G(s, t)^{(p+q) / q} \\
\leq & \frac{q}{2(p+q)}\left[F(s, t)^{2(p+q) / q}+G(s, t)^{2(p+q) / q}\right] .
\end{aligned}
$$

Now by Hölder's inequality,

$$
\begin{aligned}
F(s, t)^{2(p+q) / q} & =\left[\int_{a}^{s} \int_{c}^{t} r^{-q /(2(p+q))}(r w)^{q /(2(p+q))}\left|f_{12}\right|^{q} d v d u\right]^{2(p+q) / q} \\
& \leq\left[\int_{a}^{s} \int_{c}^{t} r^{-q /(2 p+q)} d v d u\right]^{(2 p+q) / q} \cdot\left[\int_{a}^{s} \int_{c}^{t} r w\left|f_{12}\right|^{2(p+q)} d v d u\right] \\
& \leq[(s-a)(t-c)]^{2 p / q} \cdot \int_{a}^{s} \int_{c}^{t} r^{-1} d v d u \cdot \int_{a}^{s} \int_{c}^{t} r w\left|f_{12}\right|^{2(p+q)} d v d u
\end{aligned}
$$


and similarly,

$$
G(s, t)^{2(p+q) / q} \leq[(s-a)(t-c)]^{2 p / q} \cdot \int_{a}^{s} \int_{c}^{t} r^{-1} d v d u \cdot \int_{a}^{s} \int_{c}^{t} r w\left|g_{12}\right|^{2(p+q)} d v d u .
$$

The lemma now follows by plugging these back into (2).

Q.E.D.

Proof of Theorem 1 : Since $w$ is nonincreasing in each variable, by Hölder's inequality we have

$$
\begin{aligned}
|f(s, t)| & \leq \int_{a}^{s}\left|f_{1}(u, t)\right| d u \\
& \leq \int_{a}^{s} \int_{c}^{t}\left|f_{12}(u, v)\right| d v d u \\
& \leq w(s, t)^{-1 /(2(p+q))} \int_{a}^{s} \int_{c}^{t} w(u, v)^{1 /(2(p+q))}\left|f_{12}(u, v)\right| d v d u \\
& \leq w(s, t)^{-1 /(2(p+q))}[(s-a)(t-c)]^{(q-1) / q} F(s, t)^{1 / q}
\end{aligned}
$$

and similarly,

$$
|g(s, t)| \leq w(s, t)^{-1 /(2(p+q))}[(s-a)(t-c)]^{(q-1) / q} G(s, t)^{1 / q}
$$

for any $(s, t) \in[a, b] \times[c, d]$. Hence by lemma 1 ,

$$
\begin{aligned}
& \int_{a}^{b} \int_{c}^{d} w|f g|^{p}\left(\left|f g_{12}\right|^{q}+\left|f_{12} g\right|^{q}\right) d t d s \\
\leq & \int_{a}^{b} \int_{c}^{d}[(s-a)(t-c)]^{(2 p+q)(q-1) / q} w^{q /(2(p+q))}(F G)^{p / q}\left(F\left|g_{12}\right|^{q}+G\left|f_{12}\right|^{q}\right) d t d s \\
\leq & {[(b-a)(d-c)]^{(2 p+q)(q-1) / q} \int_{a}^{b} \int_{c}^{d} w^{q /(2(p+q))}(F G)^{p / q}\left(F\left|g_{12}\right|^{q}+G\left|f_{12}\right|^{q}\right) d t d s } \\
\leq & \frac{q}{2(p+q)}[(b-a)(d-c)]^{2 p+q-1} \cdot \int_{a}^{b} \int_{c}^{d} r^{-1} d v d u \\
& \int_{a}^{b} \int_{c}^{d} r w\left(\left|f_{12}\right|^{2(p+q)}+\left|g_{12}\right|^{2(p+q)}\right) d t d s .
\end{aligned}
$$

Proof of Theorem 2: Since $0<A \leq w \leq B$ on $[a, b] \times[c, d]$, by Hölder's inequality we have,

$$
\begin{aligned}
|f(s, t)| & \leq \int_{a}^{s} \int_{c}^{t}\left|f_{12}(u, v)\right| d v d u \\
& \leq A^{-1 /(2(p+q))} \int_{a}^{s} \int_{c}^{t} w(u, v)^{1 /(2(p+q))}\left|f_{12}(u, v)\right| d v d u \\
& \leq A^{-1 /(2(p+q))}[(s-a)(t-c)]^{(q-1) / q} F(s, t)^{1 / q}
\end{aligned}
$$


and similarly,

$$
|g(s, t)| \leq A^{-1 /(2(p+q))}[(s-a)(t-c)]^{(q-1) / q} G(s, t)^{1 / q}
$$

for any $(s, t) \in[a, b] \times[c, d]$. Hence by lemma 1 ,

$$
\begin{aligned}
& \int_{a}^{b} \int_{c}^{d} w|f g|^{p}\left(\left|f g_{12}\right|^{q}+\left|f_{12} g\right|^{q}\right) d t d s \\
\leq & \int_{a}^{b} \int_{c}^{d} A^{-(2 p+q) /(2(p+q))}[(s-a)(t-c)]^{(2 p+q)(q-1) / q} \\
& w(F G)^{p / q}\left(F\left|g_{12}\right|^{q}+G\left|f_{12}\right|^{q}\right) d t d s \\
\leq & {[(b-a)(d-c)]^{(2 p+q)(q-1) / q}\left(\frac{B}{A}\right)^{(2 p+q) /(2(p+q))} } \\
& \int_{a}^{b} \int_{c}^{d} w^{q /(2(p+q))}(F G)^{p / q}\left(F\left|g_{12}\right|^{q}+G\left|f_{12}\right|^{q}\right) d t d s \\
\leq & \frac{q}{2(p+q)}[(b-a)(d-c)]^{2 p+q-1}\left(\frac{B}{A}\right)^{(2 p+q) /(2(p+q))} \int_{a}^{b} \int_{c}^{d} r^{-1} d t d s \\
& \times \int_{a}^{b} \int_{c}^{d} r w\left(\left|f_{12}\right|^{2(p+q)}+\left|g_{12}\right|^{2(p+q)}\right) d t d s .
\end{aligned}
$$

Proof of Theorem 3. We first divide the rectangle $[a, b] \times[c, d]$ into four rectangles $[a,(a+b) / 2] \times[c,(c+d) / 2],[a,(a+b) / 2] \times[(c+d) / 2, d],[(a+b) / 2, b] \times[c,(c+d) / 2]$, and $[(a+b) / 2, b] \times[(c+d) / 2, d]$. By theorem 2, inequality $(1)$ holds when restricted to the rectangle $[a,(a+b) / 2] \times[c,(c+d) / 2]$. Now by analogous statements of theorem 2 (with appropriate choices of the functions $F$ and $G$ ), it is readily seen that inequality (1) also holds when restricted to the other three rectangles. Hence the theorem.

By putting $r=w^{-1}$ in theorems 1,2 , and 3 , respectively, we have

Corollary 1. If $f(a, t)=g(a, t)=f_{1}(s, c)=g_{1}(s, c)=0$ for all $(s, t) \in[a, b] \times[c, d]$ and if $w(s, t)$ is nonincreasing in each variable, then

$$
\begin{aligned}
& \int_{a}^{b} \int_{c}^{d} w|f g|^{p}\left(\left|f g_{12}\right|^{q}+\left|f_{12} g\right|^{q}\right) d t d s \\
\leq & \frac{q}{2(p+q)}[(b-a)(d-c)]^{2 p+q-1} \int_{a}^{b} \int_{c}^{d} w d t d s \int_{a}^{b} \int_{c}^{d}\left(\left|f_{12}\right|^{2(p+q)}+\left|g_{12}\right|^{2(p+q)}\right) d t d s
\end{aligned}
$$

for any real numbers $p \geq 0, q \geq 1$.

Corollary 2. If $f(a, t)=g(a, t)=f_{1}(s, c)=g_{1}(s, c)=0$ and $0<A \leq w(s, t) \leq B$ 
for all $(s, t) \in[a, b] \times[c, d]$, then

$$
\begin{aligned}
& \int_{a}^{b} \int_{c}^{d} w|f g|^{p}\left(\left|f g_{12}\right|^{q}+\left|f_{12} g\right|^{q}\right) d t d s \\
\leq & \frac{q}{2(p+q)}[(b-a)(d-c)]^{2 p+q-1}\left(\frac{B}{A}\right)^{(2 p+q) /(2(p+q))} \int_{a}^{b} \int_{c}^{d} w d t d s \\
& \times \int_{a}^{b} \int_{c}^{d}\left(\left|f_{12}\right|^{2(p+q)}+\left|g_{12}\right|^{2(p+q)}\right) d t d s
\end{aligned}
$$

for any real numbers $p \geq 0, q \geq 1$.

Corollary 3. If $f(a, t)=g(a, t)=f(b, t)=g(b, t)=f_{1}(s, c)=g_{1}(s, c)=f_{1}(s, d)=$ $g_{1}(s, d)=0$ and $0<A \leq w(s, t) \leq B$ for all $(s, t) \in[a, b] \times[c, d]$, then

$$
\begin{aligned}
& \int_{a}^{b} \int_{c}^{d} w|f g|^{p}\left(\left|f g_{12}\right|^{q}+\left|f_{12} g\right|^{q}\right) d t d s \\
\leq & \frac{q}{2(p+q)}\left[\frac{(b-a)(d-c)}{4}\right]^{2 p+q-1}\left(\frac{B}{A}\right)^{(2 p+q) /(2(p+q))} \cdot M \\
& \times \int_{a}^{b} \int_{c}^{d}\left(\left|f_{12}\right|^{2(p+q)}+\left|g_{12}\right|^{2(p+q)}\right) d t d s
\end{aligned}
$$

for any real numbers $p \geq 0, q \geq 1$, where

$$
\begin{aligned}
M:=\max \{ & \int_{a}^{(a+b) / 2} \int_{c}^{(c+d) / 2} w d t d s, \int_{a}^{(a+b) / 2} \int_{(c+d) / 2}^{d} w d t d s, \\
& \left.\int_{(a+b) / 2}^{b} \int_{c}^{(c+d) / 2} w d t d s, \int_{(a+b) / 2}^{b} \int_{(c+d) / 2}^{d} w d t d s\right\} .
\end{aligned}
$$

By putting $r \equiv 1$ in theorems 1,2 , and 3 , respectively, we have

Corollary 4. If $f(a, t)=g(a, t)=f_{1}(s, c)=g_{1}(s, c)=0$ for all $(s, t) \in[a, b] \times[c, d]$ and if $w(s, t)$ is nonincreasing in each variable, then

$$
\begin{aligned}
& \int_{a}^{b} \int_{c}^{d} w|f g|^{p}\left(\left|f g_{12}\right|^{q}+\left|f_{12} g\right|^{q}\right) d t d s \\
\leq & \frac{q}{2(p+q)}[(b-a)(d-c)]^{2 p+q} \int_{a}^{b} \int_{c}^{d} w\left(\left|f_{12}\right|^{2(p+q)}+\left|g_{12}\right|^{2(p+q)}\right) d t d s
\end{aligned}
$$

for any real numbers $p \geq 0, q \geq 1$.

Corollairy 5: If $f(a, t)=g(a, t)=f_{1}(s, c)=g_{1}(s, c)=0$ and $0<A \leq w(s, t) \leq B$ 
for all $(s, t) \in[a, b] \times[c, d]$, then

$$
\begin{aligned}
& \int_{a}^{b} \int_{c}^{d} w|f g|^{p}\left(\left|f g_{12}\right|^{q}+\left|f_{12} g\right|^{q}\right) d t d s \\
\leq & \frac{q}{2(p+q)}[(b-a)(d-c)]^{2 p+q}\left(\frac{B}{A}\right)^{(2 p+q) /(2(p+q))} \\
& \times \int_{a}^{b} \int_{c}^{d} w\left(\left|f_{12}\right|^{2(p+q)}+\left|g_{12}\right|^{2(p+q)}\right) d t d s
\end{aligned}
$$

for any real numbers $p \geq 0, q \geq 1$.

Corollary 6. If $f(a, t)=g(a, t)=f(b, t)=g(b, t)=f_{1}(s, c)=g_{1}(s, c)=f_{1}(s, d)=$ $g_{1}(s, d)=0$ and $0<A \leq w(s, t) \leq B$ for all $(s, t) \in[a, b] \times[c, d]$, then

$$
\begin{aligned}
& \int_{a}^{b} \int_{c}^{d} w|f g|^{p}\left(\left|f g_{12}\right|^{q}+\left|f_{12} g\right|^{q}\right) d t d s \\
\leq & \frac{q}{2(p+q)}\left[\frac{(b-a)(d-c)}{4}\right]^{2 p+q}\left(\frac{B}{A}\right)^{(2 p+q) /(2(p+q))} \\
& \times \int_{a}^{b} \int_{c}^{d} w\left(\left|f_{12}\right|^{2(p+q)}+\left|g_{12}\right|^{2(p+q)}\right) d t d s
\end{aligned}
$$

for any real numbers $p \geq 0, q \geq 1$.

Notice that corollaries 4,5 , and 6 are exactly theorems 1,2 , and 3 , respectively, in [3]. In general, one would wish that the annoying term $(B / A)^{(2 p+q) /(2(p+q))}$ in theorems 2 and 3 could be removed. In fact, in [1] it was claimed that this could be done for theorem 3 by requiring $w$ to be a positive bounded function which is nonincreasing in each variable. However, the proof there contained several crucial mistakes. As a matter of fact, the following examples show that in general one could not remove the term $(B / A)^{(2 p+q) /(2(p+q))}$ in either theorem 2 or theorem 3 .

Example $\mathbb{1}$. Let $f(s, t)=g(s, t)=$ st on $[0,1] \times[0,1], p=0$, and $r=q=1$. Then

$$
\int_{a}^{b} \int_{c}^{d} w|f g|^{p}\left(\left|f g_{12}\right|^{q}+\left|f_{12} g\right|^{q}\right) d t d s=2 \int_{0}^{1} \int_{0}^{1} w \cdot s t d t d s
$$

while

$$
\frac{q}{2(p+q)}[(b-a)(d-c)]^{2 p+q} \int_{a}^{b} \int_{c}^{d} w\left(\left|f_{12}\right|^{2(p+q)}+\left|g_{12}\right|^{2(p+q)}\right) d t d s=\int_{0}^{1} \int_{0}^{1} w d t d s .
$$

Observe that any positive continuous weight function $w(s, t)$ which is large in, say $[0.8,1] \times[0.8,1]$ and small elsewhere will make $(3)>(4)$. 'Thus in general the term $(B / A)^{(2 p+q) /(2(p+q))}$ in theorem 2 is not removable. 
Notice that example 1 also shows that the nonincreasing property of the weight function $w$ in theorem 1 is essential.

Example 2. Let $f(s, t)=g(s, t)=\operatorname{st}(1-s)(1-t)$ on $[0,1] \times[0,1], p=0$, and $q=1$. Then

$$
\begin{aligned}
& \int_{a}^{b} \int_{c}^{d} w|f g|^{p}\left(\left|f g_{12}\right|^{q}+\left|f_{12} g\right|^{q}\right) d t d s \\
= & 2 \int_{0}^{1} \int_{0}^{1} w \cdot s t(1-s)(1-t)|1-2 s||1-2 t| d t d s,
\end{aligned}
$$

while

$$
\begin{aligned}
& \frac{q}{2(p+q)}\left[\frac{(b-a)(d-c)}{4}\right]^{2 p+q} \int_{a}^{b} \int_{c}^{d} w\left(\left|f_{12}\right|^{2(p+q)}+\left|g_{12}\right|^{2(p+q)}\right) d t d s \\
= & \frac{1}{4} \int_{0}^{1} \int_{0}^{1} w|1-2 s|^{2}|1-2 t|^{2} d t d s .
\end{aligned}
$$

Now it is easy to check that in $D=[0.4,0.6] \times[0.4,0.6]$, we have $s(1-s)>|1-2 s|$, $t(1-t)>|1-2 t|$. Thus for any positive continuous weight function $w(s, t)$ which is sufficiently large in $D$ and small elsewhere, we have $(5)>(6)$. Hence in general the term $(B / A)^{(2 p+q) /(2(p+q))}$ in theorem 3 is essential.

\section{References}

[1] Chen, M.S. and Lin, C.T., "A Note on Opial's Inequality," Chinese J. Math. 14, no.1 (1986) 53-58.

[2] Cheung, W.S., "On Opial-Type Inequalities in One Variable," preprint.

[3] Cheung, W.S., "On Opial-type Inequalities in Two Variables," to appear in Aequationes Mathematicae.

[4] Das, K.M., "An Inequality Similar to Opial's Inequality," Proc. Amer. Math. Soc. 22 (1969) 258261.

[5] Opial, Z., "Zur Une Inégalite", Ann. Polon. Math. 8 (1960) 29-32.

[6] Pachpatte, B.G., "On Opial-type Inequalties in Two Independent Variables," Proc. Royal Soc. Edinburgh 100 (1985) 263-270.

[7] Willet, D., "The Existence-Uniqueness Theorem for an $n$th Order Linear Ordinary Differential Equation," Amer. Math. Monthly 75 (1968) 174-178.

[8] Yang, G.S., "A Note on an Inequality Similar to Opial Inequality," Tamkang J. Math. 18, No.4 (1987) 101-104. 